\title{
Instrumentos económicos para la gestión de residuos de envases en Ecuador.
}

Economic instruments for the management of packaging waste in Ecuador.

Juan Pinos ${ }^{1}$, Ignasi Puig Ventosa ${ }^{2}$, Fernanda Banegas ${ }^{3}$, Fanny Quezada ${ }^{4}$, Gabriela Delgado ${ }^{5}$, Nataly Orellana ${ }^{6}$, Silvia Saquisilíi ${ }^{7}$ Toa Quindi ${ }^{8}$, Gustavo Chacón ${ }^{9}$

Recibido: 12-12-2017 / Revisado: 17-02-2018 Aceptado: 03-03-2018 / Publicado: 01-04-2018

\begin{abstract}
.
DOI: https://doi.org/10.33262/cienciadigital.v2i2.77

This paper aims at presenting different possible economic instruments to improve packaging waste management in Ecuador. The paper begins by presenting the current situation of solid waste management in Ecuador, focusing on packaging waste and on the Refundable Tax on Single Use Plastic Bottles in particular. Next, the state-of-theart of economic instruments applied to managing packaging waste is presented; particularly: the extended producer responsibility, environmental taxes and depositrefund schemes are discussed. The paper also evaluates possible applications for these instruments in Ecuador and proposes actions under the existing legal framework and the current system of waste management.
\end{abstract}

Keywords: Ecuador, Packaging, Environmental Taxes, Extended Producer Responsibility, Waste, Deposit-Refund Schemes.

\footnotetext{
${ }^{1}$ Maestría en Gestión Ambiental, Universidad del Azuay, Cuenca, Ecuador. juan.pinosf@gmail.com

${ }^{2}$ ENT Environment and Management, Barcelona, España. ipuig@ent.cat

${ }^{3}$ Maestría en Gestión Ambiental, Universidad del Azuay, Cuenca, Ecuador. fernanda.banegas90@ucuenca.ec

${ }^{4}$ Maestría en Gestión Ambiental, Universidad del Azuay, Cuenca, Ecuador. fannyquezada0220@gmail.com

${ }^{5}$ Maestría en Gestión Ambiental, Universidad del Azuay, Cuenca, Ecuador. gabbycha_99@hotmail.com

${ }^{6}$ Maestría en Gestión Ambiental, Universidad del Azuay, Cuenca, Ecuador. naty_orellana@hotmail.es

${ }^{7}$ Maestría en Gestión Ambiental, Universidad del Azuay, Cuenca, Ecuador. s.gsilvia04@gmail.com

${ }^{8}$ Maestría en Gestión Ambiental, Universidad del Azuay, Cuenca, Ecuador. toa_q@yahoo.es

${ }^{9}$ Escuela de Biología, Ecología y Gestión, Universidad del Azuay, Ecuador. gchacon@uazuay.edu.ec
} 


\section{Resumen.}

Este artículo tiene por objetivo presentar los posibles instrumentos económicos para mejorar la gestión de residuos de envases en Ecuador. El artículo empieza presentando la gestión de residuos sólidos urbanos en Ecuador, focalizando en la fracción envases y, en particular, el Impuesto Redimible a las Botellas Plásticas No Retornables. A continuación, se presenta el estado del arte internacional en materia de instrumentos económicos aplicados a la gestión de envases, singularmente: esquemas de responsabilidad extendida del productor, impuestos y sistemas de depósito, devolución y retorno. El artículo valora la aplicación de estos instrumentos en Ecuador, y formula propuestas considerando el marco legal y la gestión preexistente.

Palabras Claves: Ecuador, Envases, Impuestos Ambientales, Responsabilidad Extendida del Productor, Residuos, Sistemas de Depósito, Devolución y Retorno.

\section{Introducción.}

La gestión de residuos sólidos urbanos (RSU) en Ecuador ha ido evolucionando a la par de lo que ha ido exigiendo el crecimiento demográfico, la densificación en zonas urbanas, el desarrollo del sector industrial y empresarial y el cambio en los patrones de consumo, entre otros factores. El incremento en la generación de RSU ha dificultado su gestión al demandar una alta inversión económica, tanto por parte de las administraciones como por parte de empresas privadas (Jara-Samaniego et al. 2017), resultando hasta el momento en sistemas de gestión insuficientes. En tal sentido, en Ecuador el manejo de los desechos sólidos se ha constituido en una problemática social, económica y sobre todo ambiental para los gobiernos municipales, que son responsables directos de su gestión (Ruiz et al. 2015). Esta situación favorece las prácticas inadecuadas de manejo de RSU, como el vertido en arroyos y ríos, que producen graves problemas de contaminación en el suelo y las aguas superficiales (JaraSamaniego et al. 2017). Como resultado de ello se presentan afecciones a la salud humana y al medio ambiente en todos los países en vías desarrollo (Guerrero et al. 2012, Triassi et al. 2015), que se concretan también en Ecuador (Cevallos et al. 2015, Jara-Samaniego et al. 2017). La presente investigación se focaliza en la gestión de una parte de los residuos: los envases domésticos y comerciales -tales como botellas plásticas, latas, botellas de vidrio, entre otros-. Pese a no ser considerados residuos peligrosos, tienen un impacto ambiental elevado puesto que su uso cotidiano hace que la generación de este tipo de residuo se genere de manera masiva y continua (Téllez 2012), con la singular problemática de su dispersión por el medio terrestre y marino. Hasta la fecha no se ha reportado una investigación sobre la gestión integral de los envases en Ecuador. 
El presente artículo aborda los siguientes aspectos: 1) el contexto legal que existe en Ecuador en relación a la gestión de los residuos sólidos y, en particular, de los envases; 2) los modelos de gestión existentes en el país; 3) una estimación de los envases - de uso doméstico y comercial- puestos en el mercado, y las responsabilidades que tienen los productores en la circulación y recolección de los envases a lo largo de su ciclo de vida; 4) una breve descripción del estado del arte de los instrumentos económicos aplicados a la gestión de envases en otros países; y 5) la formulación de propuestas de instrumentos de este tipo para mejorar su gestión en Ecuador. Específicamente, el artículo se centra en las políticas públicas de carácter económico y fiscal para mejorar la gestión de residuos de envases.

\section{Marco legal ecuatoriano en materia de residuos.}

La Constitución del Ecuador (2008) mediante los artículos 14 y 15, reconoce el derecho de la población a vivir en un ambiente sano y ecológicamente equilibrado (Sumak Kawsay), declarando de interés público la preservación del ambiente, la conservación de los ecosistemas, la biodiversidad, la prevención del daño ambiental y la recuperación de los espacios naturales degradados, para lo cual deberá promover en el sector público y privado el uso de tecnologías ambientalmente limpias y de energías alternativas no contaminantes y de bajo impacto. La competencia exclusiva del Estado sobre las políticas económica, tributaria, aduanera, arancelaria, fiscal y monetaria; expresada en el artículo 261 de la Constitución, nos muestra plenamente la libertad que tiene el Estado para intervenir directamente en las políticas nacionales. Por ejemplo, la implementación de diferentes mecanismos económicos y financieros que promuevan el uso de tecnologías ambientalmente limpias.

La prestación de los servicios públicos de manejo de RSU es competencia exclusiva de los Gobiernos Autónomos Descentralizados Municipales (GADs), como se expresa en el artículo 264 de la Constitución, y en los artículos 55 y 137 del Código Orgánico de Organización Territorial, Autonomía y Descentralización (COOTAD). Sin embargo, esta competencia deberá seguir los lineamientos emitidos por la Autoridad Ambiental Nacional, en este caso por el Ministerio del Ambiente (MAE).

En el artículo 9 del Código Orgánico del Ambiente (COA) (Suplemento Registro Oficial No 983 emitido el 12 de abril de 2017) en su Título II de los Derechos, Deberes y Principios Ambientales expone que "los principios ambientales deberán ser reconocidos e incorporados en toda manifestación de la Administración pública". En relación a la gestión de residuos, se reconoce el principio de responsabilidad integral, es decir, para toda actividad que genere o pueda generar impactos ambientales, en todas las fases de la actividad, esto es desde su generación hasta su disposición final. Se incluye la aplicación del principio ambiental "de la cuna a la tumba" y "el que contamina paga", mediante la incorporación en los costos de 
producción de todas las medidas ambientales necesarias (ya sea para: prevenir, evitar o reducir) a fin de precautelar cualquier tipo de contaminación en el medio ambiente.

El COA en su artículo 233 sobre la Aplicación de la Responsabilidad Extendida del Productor (REP) a la gestión de residuos y desechos no peligrosos, peligrosos y especiales, menciona que "los productores tienen la responsabilidad de la gestión del producto en todo el ciclo de vida del mismo. Esta responsabilidad incluye los impactos inherentes a la selección de los materiales, del proceso de producción y el uso del producto, así como lo relativo al tratamiento o disposición final del mismo cuando se convierte en residuo o desecho luego de su vida útil o por otras circunstancias". Para el cumplimiento de la REP (que también puede referirse como responsabilidad ampliada del productor), la Autoridad Ambiental Nacional a través de normativas deberá determinar los productos sujetos a la política de REP, así como las metas y los lineamientos para la presentación del programa de gestión integral (PGI) de los residuos y desechos originados a partir del uso o consumo de los productos regulados. Sobre la REP, el Texto Unificado de Legislación Secundaria del Ministerio del Ambiente (TULSMA), en su libro VI aprobado mediante Acuerdo Ministerial 061 (emitido en mayo de 2015), artículo 50 establece que "los productores o importadores, según sea el caso, individual y colectivamente, tienen la responsabilidad de la gestión del producto a través de todo el ciclo de vida del mismo, incluyendo los impactos inherentes a la selección de los materiales, del proceso de producción de los mismos, así como los relativos al uso y disposición final de estos luego de su vida útil”. La REP se aplica en la gestión de desechos plásticos de uso agrícola (Acuerdo Ministerial 021), gestión de aparatos eléctricos y electrónicos (Acuerdo Ministerial 190) y la gestión de neumáticos usados (Acuerdo Ministerial 129).

La Ley de Fomento Ambiental y de Optimización de los Ingresos del Estado, en su capítulo II, hace referencia al denominado "Impuesto Redimible a las Botellas Plásticas No Retornables", que se expone en detalle en el apartado Impuesto redimible a las botellas plásticas no retornables.

También se cuenta con un texto normativo que promueve el reciclaje de residuos de botellas de vidrio y fabricación de botellas de vidrio con materia prima reciclada, denominado "Regulación para la Gestión de Residuos de Botellas de Vidrio en el Ecuador" (Ministerial 903 del Ministerio de Ambiente, emitido en diciembre de 2016). Esta regulación obliga al fabricante a presentar un plan para la gestión de botellas de vidrio para bebidas alcohólicas y no alcohólicas a la Autoridad Ambiental Nacional para su aprobación, el cual deberá contener el conjunto de reglas, acciones, estrategias, procedimientos y medios dispuestos para el cumplimiento de las disposiciones sobre reciclaje de botellas. La forma de fiscalización estatal será por medio de la presentación de un informe anual por parte del ente generador. 
Finalmente, el Objetivo 3 del Plan Nacional de Desarrollo 2017-2021 es "Garantizar los derechos de la naturaleza para las actuales y futuras generaciones", dentro de este marco se establecen las metas a 2021: 1) Incrementar del 70,3\% al 80\% los residuos sólidos no peligrosos con disposición final adecuada a 2021 y 2) Incrementar del 17\% al 35\% los residuos sólidos reciclados en relación al total de residuos generados, hasta 2021 (SENPLADES 2017).

\section{Introducción a la gestión de residuos en Ecuador.}

A nivel nacional, los GADs son los responsables directos del manejo y disposición de los RSU, sin embargo, han presentado una baja capacidad de gestión en este tema. La mayor parte de los GADs crearon unidades administrativas para proveer el servicio de gestión de RSU bajo la dependencia jerárquica de diferentes direcciones (p.e. Departamento de Gestión Ambiental, Departamento de Saneamiento e Higiene, etc.), en otros casos a través de las comisarías municipales que tienen una débil imagen institucional y no cuentan con autonomía administrativa ni financiera (MAE 2015a).

Importantes problemas sanitarios se presentaron en el país debido a la insuficiente gestión de los RSU, por lo que en el año 2002 la Organización Panamericana de la Salud (OPS) auspicia el estudio "Análisis Sectorial de Residuos Sólidos del Ecuador", cuyo propósito estratégico se basó en el apoyo al desarrollo de la gestión de los desechos desde un enfoque sistemático, multidisciplinario e intersectorial (MAE 2015a). Sin embargo, no se establecieron indicadores como línea base que permitan medir la eficiencia de la aplicación del estudio o de diferentes estrategias auspiciadas por el Estado.

Desde el año 2002 hasta el 2010 la situación a nivel nacional no había variado significativamente. De un total de 221 municipios, 160 disponían sus desechos en botaderos a cielo abierto y los restantes 61 municipios en sitios de disposición final parcialmente controlados (estos últimos realizan el manejo con insuficientes criterios técnicos) (MAE 2015a). Debido a esta problemática ambiental, a partir de 2009 el MAE empezó a regular y controlar mediante procesos administrativos a los municipios en temas de gestión de residuos.

Con estos antecedentes, en 2010 el Estado mediante el MAE aprobó el Programa Nacional para la Gestión Integral de Desechos Sólidos (PNGIDS). El PNGIDS fue concebido con el objetivo primordial de impulsar la gestión de los residuos sólidos en los municipios del Ecuador mediante un enfoque integral y sostenible. La finalidad del PNGIDS es disminuir la contaminación ambiental, mejorando la calidad de vida de los ciudadanos e impulsando la conservación de los ecosistemas; a través de estrategias, planes y actividades de capacitación, sensibilización y estímulo a los diferentes actores relacionados. Se establecieron metas para el año 2017, entre ellas que el 70\% de los residuos sólidos generados a nivel nacional se dispongan en rellenos sanitarios y eliminar por completo los botaderos a cielo abierto. 
En materia de gestión de residuos sólidos para el año 2015 según el Instituto Nacional de Estadística y Censos (INEC) junto la Asociación de Municipalidades Ecuatorianas (AME); 184 municipios poseen gestión directa, 20 mediantes empresas públicas mancomunadas, 12 a través de empresas públicas y 5 mediante mancomunidad (INEC-AME 2015). En 2015 de los 221 GADs municipales, 83 iniciaron y/o mantuvieron procesos de separación en la fuente de los residuos sólidos que representa el 38\% del total a nivel nacional. En adición, 161 GADs municipales realización caracterización de residuos sólidos urbanos, obteniendo 59\% de residuos orgánicos y $41 \%$ de residuos inorgánicos. En cuanto a la disposición final por parte de los GADs Municipales se obtuvo para el 2015 que 51 lo hacían en botaderos a cielo abierto, 39 en botaderos controlados, 38 en celdas emergentes y 93 en rellenos sanitarios. Concretamente, para Ecuador se determinó que la generación de residuos sólidos urbanos promedio es de 0,58 kg/hab/día (AME-INEC 2015).

Según el PNGIDS el servicio de recolección de residuos sólidos tiene una cobertura nacional promedio del 84,2\% en las áreas urbanas y del 54,1\% en el área rural. Además, el 73,4\% de los vehículos de recolección del país son compactadores (MAE 2015a). En 2016, la generación de residuos en Ecuador era de 4,06 millones de toneladas métricas al año y una generación per cápita de $0,74 \mathrm{~kg}$ (INEC 2016), que es una estimación diferente de la anteriormente presentada y que da cuenta de las incertidumbres estadísticas existentes.

Entre las ciudades que más se destacan sobre el manejo de RSU se encuentran Cuenca, Quito y Loja. Cuenca recicla mensualmente aproximadamente 102 toneladas de basura involucrando al $40 \%$ de su población, a pesar de ser la ciudad modelo en Ecuador, la gestión del reciclaje se ve aún insuficiente si se compara con las 490 toneladas de basura no reciclable que se disponen diariamente en el relleno sanitario (la generación per cápita de RSU en Cuenca es de 0,93 kg/hab/día); en adición, cabe resaltar que el relleno sanitario de Cuenca recibe también los RSU de ciertos cantones aledaños que no disponen de sistemas de disposición final de la basura. La ciudad de Cuenca es seguida por ciudades como Quito y Loja en cuanto a la recuperación de basura reciclada (Ruiz et al. 2015). A nivel nacional, la recuperación de RSU corresponde a 53,9\% de residuos orgánicos, seguido de residuos inorgánicos: $24,4 \%$ de cartón y papel, 10,8\% de plástico, 6,5\% de metales y chatarra y 4,4\% de vidrio (Solíz 2014). De los 241 cantones que conforman el país, el 45,5\% realiza alguna actividad de recuperación de residuos inorgánicos (Solíz 2014). La generación de RSU varía en cantidad y composición entre ciudades, en relación al número de habitantes y condiciones climáticas en las distintas regiones de Ecuador. Por lo tanto, los planes y estrategias de gestión de residuos reciclables varían entre administraciones.

Se constata que es notable el avance en materia de Gestión Integral de Residuos Sólidos en Ecuador, tanto en la normativa nacional como en su aplicación, sin embargo, a pesar que los GADs Municipales han desarrollado mecanismos para realizar una gestión diferenciada, los avances en el territorio no han sido todos los necesarios. 


\section{La gestión actual de envases en Ecuador.}

Actualmente se recicla el 19\% de las 105.504 toneladas de botellas y envases de vidrio puestas en el mercado en el país (MAE 2015b). Para la gestión de envases de vidrio, según el artículo 6 de los mecanismos de gestión del residuo de botellas de vidrio del Suplemento Registro Oficial No 903 (emitido en diciembre de 2016), los fabricantes de botellas de vidrio (persona que fabrique botellas de vidrio para bebidas alcohólicas y no alcohólicas dentro del territorio ecuatoriano) son los responsables del reciclaje del residuo del vidrio de postconsumo. Para ello deben implementar mecanismos de cooperación con los productores (personas que realizan el proceso de producción y embotellado de bebidas alcohólicas y no alcohólicas contenidas en vidrio para su posterior comercialización) o importadores (personas que ingresan legalmente a territorio ecuatoriano bebidas alcohólicas y no alcohólicas contenidas en botellas de vidrio), con el objetivo de generar distintos mecanismos y estrategias para promover la reducción, reutilización y reciclaje del vidrio.

Para el respectivo seguimiento y control de la gestión de envases de vidrio, los fabricantes de botellas de vidrio deberán regirse al Programa de Gestión Integral de Residuos de botellas de Vidrio de Bebidas Alcohólicas y no Alcohólicas que contiene un conjunto de reglas, acciones, estrategias, procedimientos y medios para el cumplimiento de las disposiciones establecidas en el Acuerdo Ministerial (MAE 2016).

Respecto a la gestión de los envases de cartón para bebidas, la empresa Tetra Pak en Ecuador con su proyecto "Tetra Pak Recupera y Recicla" logró recuperar 836 toneladas de estos envases (aproximadamente 67 millones de unidades), lo que equivale a un 11,9\% de todo lo vendido, lo cual respecto a 2014 representó un incremento del 44\% del volumen de recolección. La empresa se plantea para 2020 recolectar y reciclar el $40 \%$ de los envases vendidos en el país. Este programa de reciclaje ha generado 3.500 plazas de recolectores a nivel nacional, los cuales son dotados de equipos de protección necesarios para facilitar su labor de recolección por parte de la empresa (Tetra Pak 2016).

Los envases plásticos son los de mayor preocupación por el alto consumo per cápita y su lenta degradación. Por su singular problemática y por la originalidad de la medida, se expone en primer lugar Impuesto Redimible a las Botellas Plásticas no Retornables.

\section{Impuesto redimible a las botellas plásticas no retornables.}

El alto uso de las botellas plásticas no retornables es un serio problema en Ecuador. En 2014 se consumieron 98 botellas per cápita (Andrade 2016). Con la finalidad de disminuir la contaminación ambiental que estas generan por la lenta degradación de sus componentes y estimular el proceso de reciclaje, se implementó el "Impuesto Redimible a las Botellas Plásticas no Retornables - IRBPNR", el cual fue creado por medio de la Ley de Fomento Ambiental y Optimización de los Ingresos del Estado en 2011, con el objetivo de motivar a 
las personas a reciclar y así disminuir los impactos negativos, buscando un cambio de conducta entre consumidores y productores.

Por cada botella plástica gravada con este impuesto, se puede recuperar el valor pagado por concepto del impuesto que corresponde a US\$ 0,02, valor que se devolverá en su totalidad a quien recolecte, entregue y retorne las botellas, para lo cual se establecerán los respectivos mecanismos tanto para el sector privado como público para su recolección, conforme disponga el respectivo reglamento (Andrade 2016). Es decir, por medio de los establecimientos certificados por el Ministerio de Industrias y Productividad (MIPRO), en los que el Estado Ecuatoriano a través del Servicio de Rentas Internas (SRI) devuelve el valor por cada envase recuperado. Los productos lácteos y medicamentos en botellas plásticas están exentos del pago de este impuesto (MIPRO 2017).

El procedimiento de devolución del Impuesto Redimible es aplicable solo para los centros de acopio, recicladores o importadores que estén certificados por el MIPRO, quienes deben de contar con instalaciones necesarias que les permitan recibir las botellas plásticas de los consumidores o recolectores. En mayo de 2017 se encontraban certificados: 16 recicladores, 27 centros de acopio y una embotelladora; los cuales han suscrito y firmado el acuerdo de responsabilidad para la devolución del impuesto, el cual contiene seis cláusulas relacionadas con dicho impuesto (SRI 2017).

A raíz del impuesto redimible, la gestión de los envases plásticos no retornables está basada en el reciclaje, recolección y comercialización, normalmente por parte de pequeños recicladores que recolectan este material en calles, edificios, mercados, botaderos, etc. y los venden a centros de acopio, para luego ser vendidos a grandes recicladores. Según Andrade (2016), los beneficios que obtienen los recicladores de base con el IRBPNR son parciales, debido a que los mayores beneficiarios son los intermediarios y además estos evitan que los recicladores de base establezcan relaciones directas con las empresas recicladoras que existen en el país.

Se considera un sistema exitoso puesto que ha supuesto una gran reducción de las botellas plásticas tiradas en la calle o en el medio ambiente (MAE 2013). El nivel de recolección se incrementó al $81 \%$ en el primer año de vigencia del tributo, al 141,5\% en el segundo año y $121,1 \%$ en el tercer año. Sin embargo, este sistema presentó fallas, como pagos por envases ilegales provenientes de Perú y Colombia. En el año 2013, los primeros meses se recolectaron 698 millones de envases plásticos no retornables, cifra superior a los 609 millones que pagaron el impuesto, para el período 2012-2014 las recaudaciones por efecto del pago del impuesto sumaron US\$ 83,6 millones mientras que las devoluciones ascendieron a US\$ 96,3 millones (Andrade 2016). 


\section{Estado del arte internacional.}

El principio 16 de la Declaración de la Conferencia de las Naciones Unidas sobre el Medio Ambiente y Desarrollo, celebrada en 1992 en Río de Janeiro, establece que "las autoridades nacionales deberían procurar fomentar la internalización de los costos ambientales y el uso de instrumentos económicos, teniendo en cuenta el criterio de que el que contamina debe, en principio, cargar con los costos de la contaminación, teniendo debidamente en cuenta el interés público y sin distorsionar el comercio ni las inversiones internacionales".

Internacionalmente, desde finales de los años ochenta, el concepto de Responsabilidad Extendida del Productor (REP) se ha convertido en un principio establecido de la política ambiental en un creciente número de países (OECD 2016). La REP consiste en que los productores sean responsables de los impactos ambientales de sus productos en toda la cadena, desde el diseño hasta la fase post-consumo (OECD 2016). Esta responsabilidad puede ser solamente financiera o también material, pero en todo caso el hecho de ser responsable de los productos una vez convertidos en residuos debería proporcionar un incentivo para desarrollar productos que eviten desperdicios innecesarios y que puedan ser usados en operaciones de reciclaje y recuperación (Comisión Europea 2010). Por ejemplo, la REP sobre envases se encuentra establecida en la práctica totalidad de estados miembros de la Unión Europea, puesto que su aplicación se deriva de la Directiva 94/62/CE de Envases y Residuos de Envases.

Aun así, la REP puede tomar diversas formas:

- Responsabilidad material o financiera

Por un lado la responsabilidad del productor puede ser material, en el sentido que se encarga directamente de la recogida y tratamiento de los materiales, o puede ser solamente financiera, siendo en este caso la Administración quien ejerce la gestión material (o parte de ella) y los productores quienes asumen los costes.

- Responsabilidad individual o colectiva

Por otro lado, la responsabilidad puede ser individual, si cada empresa se responsabiliza solamente de lo que ella pone en el mercado, o bien colectiva, si las empresas se agrupan para gestionar colectivamente lo que todas ellas conjuntamente han puesto en el mercado.

En virtud de la responsabilidad individual del productor (IPR, por sus siglas en inglés), cada productor es responsable de la recogida y el reciclado de los residuos procedentes de sus propios productos. La característica distintiva de los IPR es que el coste de la gestión de residuos de un productor individual no se ve afectado por el comportamiento de los competidores (OECD 2016). 
Por lo referente a los sistemas de responsabilidad colectiva, existen varias características:

1. Aprovechan economías de escala y optimizan costes.

2. Los productores suelen optar por un enfoque colectivo puesto que comparte riesgos entre los competidores.

3. Existe más seguridad para las administraciones ante productores individuales que quiebren y no puedan hacer frente a su responsabilidad de gestión de los residuos (OECD 2016).

La mayoría de los programas de responsabilidad colectiva funcionan con sistemas colectivos de responsabilidad ampliada (o extendida) del productor (SCRAPs). Cuando los gobiernos imponen objetivos de recuperación (es decir, recolección o reciclado), los productores suelen establecer y financiar un SCRAP (o Sistema Integrado de Gestión -SIG- o Producer Responsibility Organisation -PRO-) para cumplir colectivamente con las obligaciones de REP. Las diferencias entre productos, mercados y políticas han inducido sistemas de responsabilidad colectiva con características muy distintas.

La responsabilidad colectiva funciona actualmente en muchos Estados miembros de la Unión Europea para la gestión de los envases. Los productores que colocan material en el mercado pagan una contribución a los SCRAPs por la recolección y el reciclaje de una cantidad relacionada de material de desecho (Comisión Europea 2010). Son los llamados sistemas Green Dot o Punto Verde, por el distintivo que diferencia a los envases sujetos al sistema.

- Con o sin depósito.

Finalmente, los envases pueden ser comercializados con o sin depósito. El sistema consiste en aplicar un depósito en el momento de la venta del producto, que se devuelve posteriormente cuando se retorna el envase vacío, para su reutilización o reciclado (OECD 2005).

La utilización de depósitos había sido tradicionalmente de uso voluntario por parte de empresas que ponían en el mercado envases reutilizables (típicamente de vidrio), pero diversos países o regiones lo han instituido como sistema colectivo de REP (p.e. Noruega o Alemania para envases de bebidas). En este caso, los responsables de la puesta en el mercado de los productos envasados cobran, en concepto de depósito, a sus sucesivos clientes hasta el consumidor final, una cantidad por cada envase. Este depósito es pagado a un ente central, que se encarga de organizar y financiar la recogida conjunta de los envases sujetos a depósito.

Estos sistemas alcanzan la mayor eficiencia en la recuperación de envases, lo cual permite disminuir el volumen de desechos enviados a los rellenos sanitarios aumentado su vida útil (Lavee 2010). 
- $\quad$ Fiscalidad.

Otra opción alternativa o complementaria a la REP, es gravar los envases con un impuesto ya sea con un carácter disuasorio (Dikgang et al. 2012, Bernal 2017) o con la finalidad de usar la recaudación para mejorar el sistema público de gestión de residuos.

Diferentes países han optado por establecer impuestos sobre los envases de un solo uso, con la finalidad de disuadir su consumo y favorecer las alternativas (p.e. en Bélgica, Croacia, Estonia, Hungría, Letonia, Noruega, Países Bajos o Polonia) (OECD 2018). La efectividad de los impuestos es mayor cuanto más elástica sea la demanda de los productos gravados. En este sentido, han sido particularmente efectivos algunos impuestos sobre las fundas plásticas de un solo uso (p.e. en Irlanda ${ }^{1}$ ), puesto que su uso es con frecuencia superfluo y sus posibilidades de sustitución elevadas.

\section{Propuestas.}

Fruto del análisis de situación anterior, en este apartado se presentan diferentes posibles instrumentos de política económica ambiental que podrían potencialmente ser aplicados a la gestión de envases en Ecuador.

\section{Sistemas colectivos de responsabilidad ampliada del productor.}

Una primera opción sería establecer un sistema de responsabilidad ampliada (o extendida) del productor (SCRAP) para envases y residuos de envases, como forma de que los productores e importadores cumplieran con su REP.

La articulación del sistema se podría realizar básicamente de dos maneras:

- El SCRAP (que sería integrado como mínimo por los productores e importadores) asume directamente la gestión, asumiendo la responsabilidad material de la recogida, transporte y tratamiento hasta su reciclaje o valorización final. La transferencia de responsabilidad de los productores/importadores al SCRAP se establecería mediante el pago de un monto basado en las ventas actuales en volumen, peso o unidades colocadas de envases en el mercado (Niza et al. 2014). Sería pertinente que estas tarifas tomasen también en cuenta el material del envase y su reciclabilidad, con el objetivo de incentivar los envases más ecológicos.

- La administración pública asume la gestión y el SCRAP la financia. La administración podría asumir la totalidad de la gestión o solamente una parte (p.e. recogida y transporte de los envases hasta plantas de titularidad del SCRAP). En el caso de participación de entidades locales en la recolección y transporte de los residuos, estas entidades

\footnotetext{
${ }^{1}$ Introducido en 2002. El tipo impositivo en 2018 es de $0,22 € /$ funda.
} 
deberían ser compensadas por su participación y por los costes que suponga este sistema. Hay dos opciones, respecto de si corresponsabilizar al SCRAP solamente de los costes adicionales del sistema de gestión de envases respecto de lo que hubiese costado con el sistema ordinario de gestión (es lo que sucede por ejemplo en España) o bien trasladar la totalidad de los costes de gestión (es decir, los costes del nuevo sistema, más la parte correspondiente a la gestión de los envases que sigan sin recogerse y tratarse separadamente). Creemos que una interpretación estricta del principio de responsabilidad extendida del productor favorece la segunda interpretación.

En ambos casos, los SCRAPS deberán ajustar su funcionamiento a las reglas propias de la figura jurídica elegida para su creación de acuerdo con la normativa reguladora. Además, dado que actuarán en régimen de monopolio o casi monopolio, deberán garantizar la participación de todos los actores en función de criterios objetivos de puesta de envases en el mercado y de sus características, así como sus derechos a la participación y a la información.

Por parte de la Administración se podría permitir la creación de SCRAPs para cada subfracción (p.e. papel, envases ligeros, vidrio) o bien optar por SCRAPs que pudieran integrar la gestión de las diferentes subfracciones. Asimismo, se podría optar por permitir un único SCRAP, o bien un único SCRAP por subfracción, o bien permitir la creación de varios de ellos posibilitando la existencia de competencia, pero al coste de una mayor complejidad administrativa y de monitoreo. En cualquier caso, el despliegue del nuevo sistema debería contar con una estrategia de comunicación acerca del programa que alcance a todos los involucrados en la cadena de producción, desde los consumidores a los responsables de la puesta en el mercado. La comunicación debe adaptar los mensajes según sean sus destinatarios (Massarutto 2014).

En el caso de Ecuador, de los dos modelos básicos presentados, nuestra recomendación sería optar por un sistema en el que los SCRAPs asumiesen directamente la gestión material de los envases, desplegando tanto la recogida selectiva como el transporte y gestión en plantas. La relativamente escasa capacidad institucional y económica de algunos GADs nos lleva a pensar que este modelo sería preferible a otro en que la recogida fuese responsabilidad de los GADs y los SCRAPs asumiesen solamente la responsabilidad financiera. Aunque también podría ser un sistema mixto, en el que algunos de los GADS con más capacidad institucional sí prestasen la recogida selectiva. Sin embargo, correspondería a la Administración la aprobación de la normativa reguladora. La propuesta de regulación debería ser impulsada por el MAE para posteriormente ser aprobada por la Asamblea Nacional. El MAE será a quien también corresponda el monitoreo del sistema para verificar el cumplimiento de la norma y el alcance de los objetivos, así como en su caso la potestad sancionadora en el ámbito de sus competencias. 
Este modelo requeriría una normativa bien concebida y un monitoreo estricto, pero permitiría el despliegue de un sistema más homogéneo en el territorio y podría beneficiarse de la mayor capacidad de inversión del sector privado. Dado que el sistema no lograría captar la totalidad de los envases, sino que una parte seguiría siendo recogida y tratada con los residuos mezclados, la parte de costes correspondiente a estos envases debería ser estimada objetivamente y trasladada a los SCRAPs para que compensasen los costes incurridos por la administración.

\section{Impuestos sobre envases.}

Como se ha comentado, diversos países han optado por gravar fiscalmente los envases de un solo uso. Tal es el caso de Ecuador, con el IRBPNR (apartado Impuesto redimible a las botellas plásticas no retornables).

En el caso de Ecuador, vemos esencialmente tres posibilidades, en caso de seguir apostando por la vía impositiva:

- La primera opción sería mejorar el impuesto actual, adoptando algunas de las lecciones de los SDDR existentes internacionalmente (ver apartado Sistemas de depósito, devolución y retorno).

- La segunda opción sería crear un impuesto generalizado sobre todos los envases de un solo uso. Sin menoscabar el posible incentivo de este impuesto a la reducción del consumo de este tipo de envases, la finalidad principal en este caso sería la obtención de recaudación para financiar públicamente los programas de recogida y tratamiento de estos envases. Sería un instrumento, por tanto, alternativo a la posible creación de sistemas colectivos de responsabilidad ampliada del productor (SCRAPS) (ver apartado Sistemas colectivos de responsabilidad ampliada del productor).

- Finalmente, existiría la opción de crear un impuesto sobre algunos envases especialmente problemáticos desde un punto de vista ambiental (p.e. fundas plásticas), como existen en diversos países (un caso exitoso se ha implementado en Colombia en 2018). En este caso, la finalidad sería sobre todo disuadir su consumo, no la recaudación que sería pequeña. En este caso, además, el impuesto no sería incompatible con la participación de estos envases en SCRAPs.

En todos los casos, pensamos que deberían ser instrumentos de regulación de ámbito estatal que deberían recaer sobre el productor y el importador de los productos envasados, tomando en cuenta en su diseño algunos de los elementos que han resultado problemáticos o fuente de fraude en el impuesto actual. En cuanto a estructuración legal, la segunda y tercera medidas se podrían articular como impuestos nuevos o como reformas del impuesto actual. En la segunda, la recaudación sería necesariamente finalista, mientras que en la tercera no, sino que recaudación podría ser finalista o ir a la caja general del Estado. 


\section{Sistemas de depósito, devolución y retorno.}

Los sistemas de depósito, devolución y retorno (SDDR) tienen la capacidad de impulsar el aprovechamiento de los recursos, debido a la alta captación de materiales -y de alta calidadque obtienen si son correctamente diseñados. Bajo este contexto podría tener sentido su implementación en Ecuador a fin de cumplir con el principio de economía circular reconocido dentro de las políticas para el logro del objetivo cuatro del Plan Nacional de Desarrollo 2017-2021 (SENPLADES 2017).

Sin embargo, antes de considerar su desarrollo es necesario tener en cuenta que el Impuesto Redimible a las Botellas Plásticas No Retornables (IRBPNR) (apartado Impuesto redimible a las botellas plásticas no retornables) existente en el país se construyó parcialmente bajo la concepción de un SDDR, ya que asocia un valor a cada unidad de producto para incentivar su recuperación. Sin embargo, a diferencia de con un SDDR, la devolución del valor no se hace directamente por parte de los consumidores sino a las embotelladoras, centros de acopio y gestores calificados, legalmente reconocidos (Andrade 2016).

A nuestro modo de ver, en Ecuador existen básicamente dos opciones:

1. Mantener la estructura legal del IRBPNR y mejorarlo con algunas de las lecciones aprendidas de los sistemas de depósito vigentes a nivel internacional.

2. Adoptar una estructura más clásica de SDDR, en la que los que ponen los envases en el mercado no pagan legalmente un impuesto posteriormente redimible, sino un depósito que se transmite en los diferentes eslabones de la cadena de distribución y consumo, y que posteriormente es recuperado por parte de cada agente en el momento de devolución del envase vacío.

En todo caso, se identifican diferentes posibilidades de mejora del sistema, con cierta independencia de su articulación legal:

- Sería recomendable establecer un operador del sistema mixto, que limitase las declaraciones fraudulentas que han realizado algunas embotelladoras bajo el mecanismo del IRBPNR.

- Sería necesario elevar el valor del impuesto/depósito por cada unidad, pues según Andrade (2016) la tarifa actual del IRBPNR, particularmente en caso de avanzar hacia un SDDR, puesto que USD 0,02 no es un factor de atracción suficiente para impulsar la devolución del envase por parte de los consumidores, a tenor de lo que se observa en otros países (Fletcher et al. 2012).

- La normativa debería garantizar que se recuperan únicamente los envases que son parte del SDDR a nivel nacional, ya que se ha demostrado que existe introducción de botellas procedentes de países vecinos donde no existen incentivos similares al 
reciclaje, tales como Perú y Colombia. Ello exigiría un etiquetaje específico de los envases sujetos al sistema.

- Se debería valorar la idoneidad de ampliar el sistema a envases de otros materiales, singularmente latas de bebidas y botellas de vidrio.

En el caso de optar por una transición desde el IRBPNR hacia un SDDR, uno de los puntos clave sería el cambio en los puntos de recogida, puesto que los consumidores deberían poder devolver los envases vacíos a la mayoría de tiendas que los distribuyen (p.e. supermercados, centros comerciales, etc.) y eventualmente a lugares públicos de elevada concurrencia si así lo prevé el modelo a desplegar (p.e. parques, centros educativos superiores, mercados municipales, etc.). Estos puntos de recolección podrían ser automáticos o manuales, pero en ambos casos deberían garantizar que se recuperan únicamente los envases involucrados dentro del SDDR. Para ello sería muy recomendable establecer un código de barras exclusivo para estos envases, el cual sería verificado y se emitiría un ticket con el valor del depósito, que podría ser canjeado o utilizado como descuento en la próxima compra. Los envases serían retirados de los lugares de acopio y transportados a un centro de acopio zonal, donde serían clasificados y compactados y finalmente enviados a recuperadores.

Considerando la estructura comercial de Ecuador, es de prever que la mayoría de puntos de recogida de envases emplearían la recogida manual, pero que la mayoría de unidades serían recogidas en los puntos de recogida automática (máquinas de vending inverso), que serían instaladas en los grandes centros de consumo. Otra opción sería admitir solamente la recogida automática, pero ello podría tener un impacto negativo sobre el pequeño comercio.

Como ya sucede actualmente con el IRBPNR, los envases no devueltos generan unos recursos. En el caso del SDDR, el sistema debe concebirse como una posible forma de aplicación de la responsabilidad extendida del productor. Así, financieramente el sistema se nutriría esencialmente de tres fuentes: la venta de los materiales recuperados, los depósitos no reclamados por parte de los consumidores que no devuelven los envases y de una posible publicidad en centros/máquinas de recepción y depósito de envases. Por regla general, estos tres conceptos son insuficientes para cubrir el coste del sistema, de modo que el remanente debería ser cubierto por las tarifas de responsabilidad extendida del productor que pagarían los productores e importadores.

Una cuestión a valorar es el coste social que este sistema podría conllevar para los recicladores de base, que son aquellos que actualmente recolectan los envases en las calles, se benefician de la devolución del impuesto redimible y por tanto perderían su ingreso económico con el SDDR, puesto que la mayoría de los envases serían devueltos directamente por el consumidor (Yildiz-Geyhan et al. 2017). Parte de la pérdida podría ser compensada por el hecho que los envases serían sujetos a un depósito presumiblemente mayor, pero esto difícilmente compensaría el impacto. En este sentido, podría habilitarse una estrategia para 
integrar parte de estos recicladores en puestos de trabajo más formales en el sistema de recogida, transporte, clasificación y procesado en planta de estos envases.

\section{Otros instrumentos económicos para favorecer el reciclaje.}

Entre otras medidas para estimular el reciclaje se podrían incluir diversas modalidades de subsidio, ya sean pagados por la recolección de materiales para reciclaje, pagados a empresas de reprocesamiento o a usuarios de materiales reciclados. También la inversión pública directa en instalaciones de tratamiento o en equipos de recogida selectiva (contenedores, camiones, campañas, etc.).

El uso de materiales reciclados también podría ser fomentado por las regulaciones que exigiesen el mínimo contenido de materiales reciclados en ciertos productos, o por impuestos sobre materiales vírgenes (OECD 2005).

\section{Conclusiones.}

- Este artículo ha abordado la gestión de residuos de envases en Ecuador y lo ha abordado a partir del estado del arte y los posibles instrumentos de carácter económico que podrían contribuir a mejorar la gestión.

- Ante la posible aplicación de estos instrumentos en Ecuador se derivan diversas conclusiones relacionadas con las tres grandes familias de instrumentos analizados: SCRAP, SDDR e impuestos.

- Por un lado, el impuesto redimible no parece compatible con el establecimiento formal de un SDDR, puesto que persiguen el mismo tipo de envases y crean incentivos parecidos. O se opta por un instrumento o por el otro. Aun así, la preexistencia del IRBPNR condiciona las acciones a tomar. Si el impuesto se desea mantener, la idea de un posible SDDR decaería formalmente si bien las lecciones de los SDDR existentes internacionalmente serían útiles para mejorar el tributo (p.e. estrategias de lucha contra el fraude, mayores importes o una mayor cobertura de materiales, como latas). Además, algunos agentes podrían habilitar sistemas manuales o automáticos de recogida similares a los de los SDDR, con el objeto de capturar envases y tramitar la correspondiente devolución. Por el contrario, si se opta por la transición hacia un SDDR más convencional, deberá reformarse la arquitectura legal, aumentarse ciertamente el importe del depósito, organizarse toda la operativa de recogida orientada especialmente a consumidores y comercios y habilitar mecanismos para minimizar el impacto sobre los pequeños recolectores que se ganan la vida con el sistema actual. Cabe señalar que el SDDR afectaría solo unos envases concretos y que por tanto otros mecanismos de recolección de desechos y de reciclaje deberían seguir funcionado para el resto de envases no sujetos. 
- Por otro lado, la posibilidad de abordar la gestión de envases mediante un impuesto generalizado sobre los mismos, podría aprovechar la estructura ya existente del impuesto redimible, debidamente ampliada. Así, todos los envases (o ciertas categorías) pagarían un impuesto que, para algunos de ellos, sería redimible (suponiendo la continuación de este instrumento) y, para el resto, serviría simplemente para financiar su gestión.

- Caso de optar por un impuesto generalizado, no tendría sentido establecer un SCRAP que asumiese material o financieramente la gestión puesto que esta sería asumida públicamente y financiada por el impuesto. Podría decirse que en este caso la REP sería asumida por la vía de un impuesto.

- Esto sería así salvo casos de envases concretos con singular problemática, como las fundas plásticas, para los que podría tener sentido un gravamen incluso si también participasen en el SCRAP. En este caso, la finalidad de este impuesto no sería recaudar para pagar su gestión - que ya sería cubierta por el SCRAP-, sino disuadir el consumo.

- Finalmente, existiría la posibilidad de optar por establecer un SCRAP para todos los envases, excepto aquellos sujetos al impuesto redimible o a un SDDR que eventualmente lo sustituyera. Como se ha dicho, en este caso nuestra recomendación preliminar sería que el SCRAP asumiese la responsabilidad material de la gestión selectiva de envases, más la responsabilidad financiera de la parte de envases que se siguiesen gestionando de forma no selectiva.

- En todos los casos, el IRBPNR supone una preexistencia importante y, eventualmente, jugaría un papel de transición muy relevante, especialmente hacia el SDDR o hacia un posible impuesto más generalizado.

- El IRBPNR requiere reformas para cumplir óptimamente con sus objetivos extra fiscales: reducir el nivel de contaminación, impulsar la renovación tecnológica y propiciar cambios en los patrones de consumo.

- Se debe crear nuevas normativas técnicas bajo el principio de la REP, estableciendo una política de post-consumo para distintos tipos de envases (Orset et al. 2017).

- Cabe decir, además, a modo de conclusión, que este artículo se ha centrado en los instrumentos económicos, pero que una política global de envases debe contener elementos de planificación y normativos que aquí no se han abordado como por ejemplo, elementos de ecodiseño o la definición de objetivos de reciclaje. Es la adecuada combinación de los diferentes elementos lo que conseguirá los resultados deseados. 


\section{Referencias bibliográficas.}

Andrade, C. G. (2016). Análisis del Impuesto Redimible a las Botellas Plásticas No Retornables en el Ecuador. Tesis de Maestría. Universidad Andina Simón Bolívar. Quito, Ecuador.

Bernal, L. F. (2017). Impuesto nacional al consumo de bolsas plásticas de utilización desechable en Colombia. Tesis de Grado. Universidad Militar Nueva Granada. Bogotá, Colombia.

Cevallos, E., Gómez, L., Roldán, A. (2015). Análisis de los problemas ambientales en el cantón la concordia, provincia santo domingo de los Tsáchilas, Ecuador. Investigación y Saberes, 4(1), 1-16.

Comisión Europea (2010). Being wise with waste: the EU's approach to waste management. Luxembourg: Publications Office of the European Union, ISBN 9789279142970.

Constitución del Ecuador (2008). Asamblea Nacional. Quito. Ecuador.

Diario El Telégrafo - DET (2014). Ecuador produjo más de 1.400 millones de botellas plásticas en 2013. Disponible en: https://www.eltelegrafo.com.ec/noticias/economia/1/ecuador-produjo-mas-de-1400-millones-de-botellas-plasticas-en-2013 [16/04/2018]

Dikgang, J., Leiman, A., Visser, M. (2012). Analysis of the plastic-bag levy in South Africa. Resources, Conservation and Recycling, 66, 59-65.

Fletcher, D., Hogg, D., von Eye, M., Elliott, T., Bendali, L. (2012). Evaluación de costes de introducción de un sistema de depósito, devolución y retorno en España. Resumen ejecutivo. Eunomia Research \& Consulting.

Guerrero, L. A., Valverde, V. R., Maas, G. (2012). Waste Management Systems' Impact on Health and Environment in Developing Countries. Linnaeus Eco-Tech.

INEC-AME (2015). Estadística de Información Ambiental Económica en Gobiernos Autónomos Descentralizados Municipales GIRS - 2015. Disponible en: http://www.ecuadorencifras.gob.ec/documentos/webinec/Encuestas_Ambientales/Gestion_Integral_de_Residuos_Solidos/Presentacion\% 20GIRS\%202015.pdf [01/04/2018]

Instituto Nacional de Estadística y Censos (INEC) (2016). Información Ambiental en Hogares. Disponible en: http://www.ecuadorencifras.gob.ec/documentos/web- 
inec/Encuestas_Ambientales/Hogares/Hogares_2016/Documento\%20tecnico.pdf [01/04/2018]

Jara-Samaniego, J., Pérez-Murcia, M. D., Bustamante, M. A., Pérez-Espinosa, A., Paredes, C., López, M., López, D., Gavilanes, I., Moral, R. (2017). Composting as sustainable strategy for municipal solid waste management in the Chimborazo Region, Ecuador: Suitability of the obtained composts for seedling production. Journal of cleaner production, 141, 1349-1358.

Lavee, D. (2010). A cost-benefit analysis of a deposit-refund program for beverage containers in Israel. Waste management, 30(2), 338-345.

Ley de Fomento Ambiental y optimización de los Ingresos del Estado (2011). Suplemento Registro Oficial $N^{\circ} 583$ de 24-nov-2011 Ecuador.

Massarutto, A. (2014). The long and winding road to resource efficiency-An interdisciplinary perspective on extended producer responsibility. Resources, Conservation and Recycling, 85, 11-21.

Ministerio de Industrias y Productividad - MIPRO (2017). Impuesto redimible a las botellas plásticas no retornables PET. Disponible en: http://www.industrias.gob.ec/impuestoredimible-a-las-botellas-plasticas-no-retornables-pet [01/04/2018]

Ministerio del Ambiente - MAE (2013). Ecuador incrementó la recolección de Botellas PET en 2012. Disponible en: http://www.ambiente.gob.ec/ecuador-incremento-larecoleccion-de-botellas-pet-en-2012/ [10/03/2018]

Ministerio del Ambiente - MAE (2015a). Programa 'PNGIDS' Ecuador. Disponible en: http://www.ambiente.gob.ec/programa-pngids-ecuador/ [10/03/2018]

Ministerio del Ambiente - MAE (2015b). Reciclaje de vidrio del país con actores estratégicos del sector. Disponible en: http://www.ambiente.gob.ec/reciclaje-de-vidrio-del-paiscon-actores-estrategicos-del-sector/ [09/03/2018]

Ministerio el Ambiente - MAE (2016). Expídese la regulación para la gestión de residuos de botellas de vidrio en el Ecuador. Acuerdo Ministerial 121 - Registro Oficial No 903.

Niza, S., Santos, E., Costa, I., Ribeiro, P., Ferrão, P. (2014). Extended producer responsibility policy in Portugal: a strategy towards improving waste management performance. Journal of cleaner production, 64, 277-287.

Organisation for Economic Co-operation and Development - OECD (2005). Analytical Framework for Evaluating the Costs and Benefits of Extended Producer Responsibility Programmes. 
Organisation for Economic Co-operation and Development - OECD (2016). Extended Producer Responsibility - Updated Guidance for Efficient Waste Management.

Organisation for Economic Co-operation and Development - OECD (2018). Database on Policy Instruments for the Environment. https://pinedatabase.oecd.org [15/04/2018]

Orset, C., Barret, N., Lemaire, A. (2017). How consumers of plastic water bottles are responding to environmental policies?. Waste Management, 61, 13-27.

Ruiz, M., Luzuriaga, H., Rodríguez, Á. (2015). El comportamiento del consumidor y la cultura de reciclaje de residuos sólidos: caso mancomunidad Patate-Pelileo. Revista digital de Medio Ambiente "Ojeando la Agenda", (36), 1.

Secretaría Nacional de Planificación y Desarrollo - SENPLADES (2017). Plan Nacional de Desarrollo 2017-2021-Toda una Vida.

Servicio de Rentas Internas - SRI (2017). Listado de Centros de Acopio, Recicladores e Importadores. Disponible en: http://www.sri.gob.ec/web/guest/listado-de-centros-deacopio-recicladores-e-importadores [10/02/2018]

Solíz, M. F. (2014). Ecología política y geografía crítica de la basura en el Ecuador: Determinación social y conflictos distributivos. Ecología Política, (47), 56-61.

Téllez, A. (2012). La complejidad de la problemática ambiental de los residuos plásticos: una aproximación al análisis narrativo de política pública en Bogotá. Tesis Doctoral. Universidad Nacional de Colombia. Bogotá, Colombia.

Tetra Pak (2016). Tetra Pak logra recuperar y reciclar cerca de 67 millones de envases puestos en el mercado. Disponible en: http://tpcomprod.azurewebsites.net:80/ec/about/newsarchive/tetra-pak-logra-recuperar-yreciclar-cerca-de-67-millones-de-envases-puestos-en-el-mercado [22/03/2018]

Triassi, M., Alfano, R., Illario, M., Nardone, A., Caporale, O., \& Montuori, P. (2015). Environmental pollution from illegal waste disposal and health effects: A review on the "Triangle of Death". International journal of environmental research and public health, 12(2), 1216-1236.

Yildiz-Geyhan, E., Altun-Çiftçioğlu, G. A., \& Kadirgan, M. A. N. (2017). Social life cycle assessment of different packaging waste collection system. Resources, Conservation and Recycling, 124, 1-12.

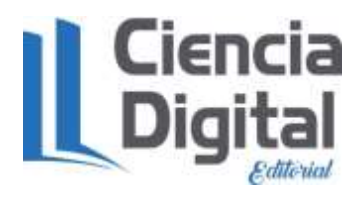




\section{Para citar el artículo indexado.}

Pinos J., Puig I., Banegas F., Quezada F., Delgado G., Orellana N., Saquisilí S., Quindi T. \& Chacón G. (2018). Instrumentos económicos para la gestión de residuos de envases en Ecuador. Revista electrónica Ciencia Digital 2(2), 123-144. Recuperado desde: http://cienciadigital.org/revistacienciadigital2/index.php/CienciaDigital/article/view/77/72

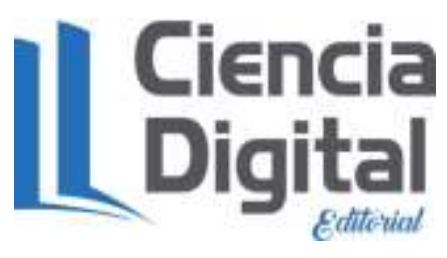

El artículo que se publica es de exclusiva responsabilidad de los autores y no necesariamente reflejan el pensamiento de la Revista Ciencia Digital.

El articulo queda en propiedad de la revista y, por tanto, su publicación parcial y/o total en otro medio tiene que ser autorizado por el director de la Revista Ciencia Digital.
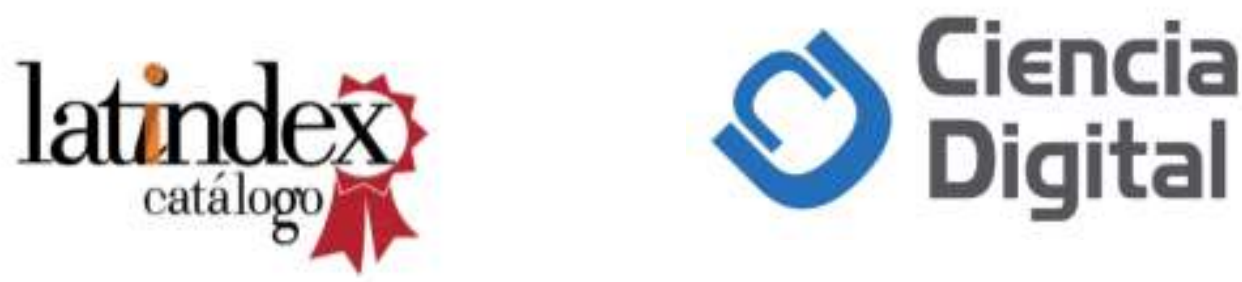